\title{
La Ley de Thirlwall: Teoría y evidencia empírica. Los casos de Méjico y Argentina en el periodo 1960-2014.
}

Thirlwalls law: theoretical and empirical evidence. The Mexican and Argentinian cases in 1960-2014.

\author{
Santiago Gabriel Manuel Capraro Rodríguez
}

\begin{abstract}
ARGENTINA
Resumen: Este trabajo investiga los determinantes teóricos y empíricos de la ley de Thirlwall para establecer si los mismos son importantes para explicar los problemas de crecimiento de México y Argentina en el periodo 1960-2014. El trabajo utiliza dos metodologías: la primera desarrolla un modelo teórico que resalta la importancia de los factores de demanda y estructura para poner freno a los procesos de crecimiento en una economía; la segunda recurre a la información histórica de las economías consideradas, y a través de modelos econométricos estima los coeficientes de la ley de Thirlwall. El principal hallazgo del trabajo es que en el caso de Argentina la ley de Thirlwall es relevante para explicar sus problemas de crecimiento, mientras que no lo es para México.
\end{abstract}

Palabras clave: crecimiento económico, balanza de pagos, ley de Thirlwall.

Abstract: This article analyses the theoretical and empirical determinants of the Thirlwall's Law. It stablishes if they are important to explain the Argentina's and Mexico's growth problems during 1960-2014. This paper utilizes two methodologists. First, it develops a theoretical model which highlights the importance of demand and structural factors as the principal obstacle to the growth process of an economy. Second, it uses historical data to estimate the relevant Thirlwall coefficients through econometric models. The most important result of this investigation is that the Thirlwall law is relevant to explain Argentina's growth problems, but it is not the case for Mexico.

Key words: economic growth; balance of payments; Thirlwall's law.

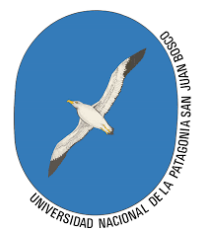

Santiago Capraro es Doctor en Economía de la Universidad Nacional Autónoma de México, Maestro en Economía de la Universidad Nacional Autónoma de México, Licenciado de Economía de la Universidad Católica Argentina. Profesor con dedicación semiexclusiva del espacio curricular de Desarrollo Económico, Universidad Nacional de la Patagonia San Juan Bosco, Facultad de Ciencias Económicas.

Contacto: santiago.capraro@gmail.com. 


\title{
Lei Thirlwall: teoria e evidência empírica.
}

Os casos do méxico e da argentina no período 1960-2014.

\begin{abstract}
Resumo: Este artigo investiga os determinantes teóricos e empíricos da lei Thirlwall, para estabelecer se eles são importantes para explicar os problemas do crescimento no México e na Argentina no período 1960-2014. O trabalho utiliza duas metodologias, um modelo teórico que enfatiza a importância da demanda e fatores estruturais para reduzir os processos de crescimento em uma economia se desenvolve em primeiro lugar. A segunda utiliza informação histórica das duas economias consideradas e através de modelos econométricos estima os coeficientes da lei de Thirlwall. A principal conclusão do trabalho é que, no caso da Argentina da lei Thirlwall é relevante para explicar os seus problemas de crescimento enquanto não é para o México.
\end{abstract}

Palavras-chave: crescimento económico; balança de pagamentos; a lei de Thirlwall.

\section{Introducción}

$\mathrm{U}$ na de las cuestiones fundamentales en la disciplina económica es explicar por qué algunas economías crecen más que otras y por qué en determinados periodos una economía acelera o disminuye su tasa de crecimiento. Cada corriente de pensamiento económico responde a este interrogante de diferente manera; por ejemplo, Thirlwall (1979) explica que:

"El enfoque neoclásico a la cuestión de las razones por las cuales algunos países crecen más que otros, [...] se concentra en el lado de la oferta de la economía, usando el concepto de la función de producción. Dada una función de producción, el crecimiento de la producción se distribuye entre el crecimiento del capital, el crecimiento del trabajo y el crecimiento de la productividad total de los factores como un residuo" (p. 45).

En la actualidad ello sigue siendo cierto y se puede agregar que se le ha dado una mayor relevancia al desarrollo del capital humano y al establecimiento de las instituciones correctas (Acemoglu y Robinson, 2012:450). La demanda efectiva continúa sin tener un papel relevante en los modelos de crecimiento neoclásico.

El principal aporte de Thirlwall (1979:44) es poner la demanda efectiva en el centro de la explicación de las diferencias en las tasas de crecimiento. Thirlwall (1979) forma parte de una línea de investigación que había sido desarrollada por algunos estructuralistas latinoamericanos; por ejemplo, vemos claros visos de la ley de Thirlwall en Furtado (1956): 
"Nuestros países no pueden seguir dependiendo de la exportación de bienes primarios para sustentar sus economías. La primera tarea que como naciones debemos llevar adelante es reorientar nuestras políticas de inversión direccionadas a la promoción de la industrialización buscando el objetivo de balancear el comercio internacional. Adicionalmente, dado que la promoción industrial genera ganancias de escala y de innovación tecnológica, promover esos objetivos será beneficioso para toda la economía" (p.68).

Incluso en Prebisch (1949:480) se pueden encontrar bases del modelo de Thirlwall, por ejemplo, cuando afirma que:

"Si no fuera suficiente el razonamiento para persuadirnos de la estrecha conexión entre el desarrollo económico y el intercambio, ciertos hechos que están ocurriendo bastarían para ponerla de manifiesto. La mayor parte de los países latinoamericanos han aumentado intensamente su actividad económica, y se encuentran en un nivel de ocupación relativamente alto si se les comparara con el anterior a la guerra. Este alto nivel de ocupación exige también elevadas importaciones, tanto de artículos de consumo, así inmediato como duradero, cuanto de materias primas y artículos de capital. Y en muchos casos, las exportaciones resultan insuficientes para satisfacer aquéllas" (p.480).

El presente trabajo tiene dos objetivos fundamentales: primero, analizar a partir de la ley de Thirlwall las diferencias en las tasas de crecimiento en el periodo 1960-2014 del producto bruto interno (PBI) de dos las principales economías de América Latina, como son México y Argentina. Segundo, analizar la incidencia de la variación de los términos del intercambio originada en el incremento del precio de las materias primas durante el periodo 2003-2014 sobre la restricción externa y, por tanto, sobre el crecimiento económico de los países analizados.

El trabajo se organiza en 4 secciones, contando la presente introducción. En la siguiente sección se analizan las bases teóricas de la ley de Thirlwall original y algunas extensiones. Luego se desarrollan modelos de cointegración para calcular la elasticidad ingreso de la demanda por importaciones de México y Argentina, con el objetivo principal de analizar si las diferencias en las tasas de crecimiento de las economías se pueden explicar por los fundamentos de la ley de Thirlwall y analizar si las economías analizadas efectivamente han encontrado su crecimiento restringido por la balanza de pagos. Finalmente se concluye. 


\section{El modelo teórico de la ley de Thirlwall}

Thirlwall $(1979,2003: 55)$ sostiene que la restricción más importante que enfrenta una economía para acelerar su tasa de crecimiento no se encuentra por el lado de la oferta, sino a la falta de demanda. Thirlwall destacó que la primera restricción que se manifiesta en una economía es la restricción externa, dificultando la aceleración de la tasa de crecimiento; una expresión de esta restricción es la imposibilidad de mantener un déficit en la cuenta corriente de la balanza de pagos (BP) en forma sostenida.

Detrás de los planteos de Thirlwall hay un tipo de cambio real constante. A partir de ese supuesto la prognosis de Thirlwall (1979) es que la tasa de crecimiento efectiva de la economía tiende a converger a una tasa de crecimiento consistente con el equilibrio de la balanza de pagos (TCCEBP), que se define más adelante. Para llegar a una expresión de la ley de Thirlwall (LT) es necesario desarrollar un modelo que contiene tres ecuaciones y tres variables endógenas:

$$
\begin{aligned}
& X_{t}=\left(\frac{E_{t} P_{t}^{*}}{P_{t}}\right)^{\eta} Y_{t}^{* \varepsilon} \text { donde } \eta>0, \varepsilon>0 \\
& M_{t}=\left(\frac{E_{t} P_{t}^{*}}{P_{t}}\right)^{-\psi} Y_{t}^{\pi} X_{t}^{\alpha} \text { donde } \psi>0, \pi>0 \\
& P_{t} X_{t}=E_{t} P_{t}^{*} M_{t}
\end{aligned}
$$

La ecuación (1) representa la demanda por exportaciones expresada en términos reales, donde $E_{t}$ es el tipo de cambio nominal (expresado como unidades de moneda nacional por unidad de moneda extranjera), $P_{t}^{*}$ es el nivel de precios internacional, $P_{t}$ es el nivel de precios de la economía local, $Y_{t}^{*}$ es el nivel de ingreso real mundial y, finalmente, $\eta$ y $\varepsilon$ son las elasticidades tipo de cambio real e ingreso de la demanda por exportaciones. La ecuación (2) representa la demanda por importaciones reales, la cual depende del tipo de cambio real definido como $Q_{t}=\frac{E_{t} P_{t}}{P_{t}}$ y del nivel de ingreso real de la economía nacional $\left(Y_{t}\right)$. Los parámetros $\psi$ y $\pi$ son las elasticidades tipo de cambio real e ingreso de la demanda por importaciones.

La ecuación (3) es el equilibrio de la cuenta corriente de la balanza de pagos (BP), la cual está expresada en moneda nacional. Las tres variables endógenas que determina el modelo son $X_{t^{\prime}}, M_{t}$ y $Y_{t^{\prime}}$ donde $Y_{t}$ no es el nivel observado del ingreso nacional real, sino aquel nivel de ingreso que es consistente con el equilibrio de la BP. El nivel de producto mundial $\left(Y_{t}\right)$ es exógeno al igual que el tipo de cambio nominal, el nivel de precios internacional y el nivel de precios nacional. Estos supuestos se ajustan a 
las características de las economías pequeñas. Una de las críticas que ha recibido este modelo es que en general ese tipo de economías enfrentan demanda por exportaciones con elasticidades precio infinita; por tanto, dado el nivel de ingreso de la economía mundial, la economía puede vender la cantidad de bienes que quiera a los precios internacionales; en particular, este parece ser el caso de una parte importante de las economías de América Latina. Recientemente se debatió este aspecto en Clavijo y Ros (2015:14), Vernengo (2015:69), Caldentey $(2015: 48,2016)$ e Ibarra (2015:41).

Si suponemos que se cumple la ecuación (1) podemos calcular la tasa de crecimiento de la economía que es consistente con el equilibrio de la BP. Con ese objetivo aplicamos logaritmos naturales a las tres ecuaciones y diferenciamos las expresiones respecto del tiempo, lo que resulta en:

(4) $x_{t}=\eta\left(e_{t}+p_{t}^{*}-p_{t}\right)+\varepsilon y_{t}^{*}$,

(5) $m_{t}=-\psi\left(e_{t}+p_{t}^{*}-p_{t}\right)+\pi y_{t}$,

(6) $p_{t}+x_{t}=e_{t}+p_{t}^{*}+m_{t}$

En cada ecuación las letras minúsculas representan la tasa de crecimiento de cada variable. Luego se introducen las ecuaciones (4) y (5) en (6) y resolviendo para la tasa de crecimiento se obtiene:

(7) $y_{t}^{B O P}=\frac{\varepsilon y_{t}^{*}+(\eta+\psi-1) q_{t}}{\pi}$

La ecuación (7) indica que la tasa de crecimiento consistente con el equilibrio de la BP $\left(y_{t}^{B O P}\right)$ depende de la tasa de crecimiento de la economía mundial $\left(y_{t}^{*}\right)$, el ratio de las elasticidades ingreso de las demandas por exportaciones e importaciones $\left(\frac{\varepsilon}{\pi}\right)$ y de la variación del tipo de cambio real multiplicada por la expresión $(\eta+\psi-1)$, que si es mayor a la unidad se conoce como condición Marshall-Lerner (Kemp, 2008). De la expresión (7) se infiere que un aumento (reducción) de la elasticidad ingreso de las exportaciones respecto a la elasticidad ingreso de las importaciones expande (contrae) la TCCEBP.

A partir de la ecuación (7) se infiere que si se cumple la condición MarshallLerner una depreciación (apreciación) incrementará (disminuirá) la TCCEBP. Thirlwall (1979) -al igual que trabajos posteriores (por ejemplo, Moreno-Brid (2003:348), Perrotini (2003:239)- hizo un fuerte supuesto acerca de la evolución del tipo real al suponer que es constante en el largo plazo, entonces $q_{t}=0$, por tanto podemos reescribir la ecuación (7) como:

(8) $y_{t}^{B O P^{\prime}}=\frac{\varepsilon}{\pi} y_{t}^{*}$ 
La ley de Thirlwall indica que en el largo plazo una economía tiende a crecer a la tasa determinada por la ecuación (8). El principal mensaje del modelo es que la tasa de crecimiento $y_{t}^{B O P^{\prime}}$ puede ser distinta de la que provoca el pleno empleo en la economía, es decir, la economía puede detener su crecimiento antes de alcanzar el pleno empleo y es en ese sentido que decimos que una economía se encuentra restringida por la BP.

A partir de las ecuaciones (8) y (4) es simple plantear que la TCCEBP es igual a la inversa de la elasticidad ingreso de las importaciones multiplicada por la tasa de crecimiento de las exportaciones, es decir:

(8) $y_{t}^{B O P^{\prime}}=\frac{1}{\pi} x_{t}$

Las ecuaciones (8) y ( $\left(8^{\prime}\right)$ reflejan que la restricción externa no se origina exclusivamente en la demanda, sino que la oferta también juega un rol importante. Claramente la restricción externa puede originarse en una baja elasticidad ingreso de la demanda por exportaciones o por una alta elasticidad ingreso de las importaciones. Además, las elasticidades ingreso de las exportaciones e importaciones dependen del tipo de productos que el país vende y compra; por tanto, el valor del cociente $\left(\frac{\varepsilon}{\pi}\right)$ es reflejo de la especialización productiva de esa economía (Abeles et al., 2013:26). Volviendo a la crítica de Clavijo y Ros (2015:38) es interesante observar que la ecuación ( $\left.8^{\prime}\right)$ se puede obtener si suponemos una tasa de crecimiento de las exportaciones determinada exógenamente, como se hace en Thirlwall y Hussain (1982:505). Es decir, a pesar de que no exista una demanda por exportaciones con pendiente negativa -como en la ecuación (1)- los resultados del modelo de Thirlwall se mantienen.

Cuando se tiene en cuenta los flujos de capitales la BP no tiene que estar necesariamente siempre equilibrada, porque los déficits se pueden financiar a través de movimiento de capital. Para estudiar ese caso debemos modificar la ecuación (3) para representar el nuevo equilibrio de la BP:

(9) $P_{t} X_{t}-P_{t} R_{t}+P_{t} F_{t}=E_{t} P_{t}^{*} M_{t}$

Donde $R_{\mathrm{t}}$ es el pago real de intereses en el periodo t y $F_{t}$ es el déficit real de la cuenta corriente. El nuevo modelo se compone de las ecuaciones (1), (2) y (9). Siguiendo a Britto y McCombie (2009) se supone que la economía puede mantener un déficit de la cuenta corriente, pero los mercados solo aceptan que sea constante en términos del nivel de producto de la economía $\left(Y_{t}\right)$. Si ese nivel se supera, los agentes del sector financiero paran repentinamente de financiar la economía. Lo anterior implica que se debe cumplir la siguiente condición: 
(10) $\frac{F_{t}}{Y_{t}}=b$ donde $b$ es constante.

Cuando se calcula la tasa de variación de las ecuaciones (1), (2), (9) y (10) y el sistema se resuelve para la tasa de crecimiento del ingreso, se obtiene la condición (11):

(11) $y_{t}^{B O P F}=\frac{\theta_{1} \varepsilon y_{t}^{*}+\left(\theta_{1} \eta+\psi-1\right) q_{t}-\theta_{2} i_{t}}{\pi-\left(1-\theta_{1}+\theta_{2}\right)}$

Donde $\theta_{1}$ es la ratio exportaciones sobre importaciones, $\theta_{2}$ es la ratio de los intereses sobre las importaciones medidas en moneda nacional y $i$ es la tasa de crecimiento del pago de intereses. Si el tipo de cambio es constante en el largo plazo, la expresión (11) puede reescribirse como:

$$
\text { (12) } y_{t}^{B O P F^{\prime}}=\frac{\theta_{1} \varepsilon y_{t}^{*}-\theta_{2} i}{\pi-\left(1-\theta_{1}+\theta_{2}\right)}
$$

Hay múltiples abordajes para estudiar si una economía se encuentra o no restringida por la BP. En el presente trabajo se comparan las elasticidades ingreso de las importaciones que surgen de las distintas expresiones de la ley de Thirlwall reseñadas en esta sección con las elasticidades ingreso de las importaciones calculadas a través de modelos de cointegración de la ecuación (2). Si coinciden se puede afirmar que la economía está restringida por la BP y que la ley de Thirlwall es una buena aproximación a la tasa de crecimiento efectiva. Si difieren se debe explicar cuáles han sido los motivos de las diferencias. En la siguiente sección se realizan los cálculos pertinentes.

\section{Estimaciones de la elasticidad ingreso de la demanda por importaciones}

En la presente sección se calculan tasas de crecimiento consistentes con el equilibrio de la balanza de pagos (TCCEBP), basadas en las distintas versiones de la ley de Thirlwall, analizadas en la sección 2 para México y Argentina en el periodo 1960-2014. Luego se comparan los resultados para establecer si las diferencias en el crecimiento de estas dos economías, tanto en relación con su propia historia como entre ellas, se pueden explicar con los fundamentos de la ley de Thirlwall. Finalmente, se estiman modelos trimestrales para el periodo 2003-2014 con el objetivo de estudiar si hubo cambios estructurales respecto al periodo original y analizar los efectos de la evolución de los términos del intercambio en el periodo 2003-2014.

Existen diversas formas de calcular la TCCEBP, y uno de los componentes básicos para hacerlo es calcular la elasticidad ingreso de la demanda de importaciones. Para ello se puede recurrir a las siguientes técnicas: 
1. LT original: $\dot{y}_{t}=\frac{\hat{\varepsilon} \hat{y}_{t}^{*}+(\hat{\eta}+\hat{\psi}-1) \dot{q}_{t}}{\hat{\pi}} \operatorname{con} \dot{q}_{t}=0$ obtenemos $\hat{\pi}=\frac{\dot{x}_{t}}{\dot{y}_{t}}$. Donde la línea sobre una variable indica que se tiene en cuenta sut valor en el periodo de estudio.

2. LT ampliada con flujos de capitales: $\dot{y}_{t}=\frac{\theta_{1} \hat{\varepsilon} \hat{y}_{t}^{*}+\left(\theta_{1} \hat{t}+\hat{\psi}-1\right) \dot{q}_{t}-\theta_{2} \dot{i}_{t}}{\hat{\pi}-\left(1-\theta_{1}+\theta_{2}\right)} \operatorname{con} \dot{q}_{t}=0$ obtenemos $\hat{\pi}=\frac{\theta_{1} \hat{\varepsilon} \hat{y}_{t}^{*}-\theta_{2} i_{t}}{\hat{x}_{t}}+\left(1-\theta_{1}+\theta_{2}\right)$. Se utilizó el valor promedio en el periodo de estudíto de $\theta_{1}$ y $\theta_{2}$ (ver la sección 2 para las definiciones de las variables).

3. Elasticidad implícita de las importaciones, calculada a través de la siguiente regresión: $y_{t}=\beta x_{t}+\epsilon_{t^{\prime}}$ donde $\beta$ es un estimador de $\frac{1}{\pi}$.

4. Estimaciones econométricas de la función de demanda de importaciones como la ecuación (2).

La tabla 1 muestra los resultados del cálculo de las elasticidades ingreso de las importaciones de las economías analizadas. Los resultados indican que las elasticidades ingreso de las importaciones consistentes con el equilibrio de la balanza de pago (EIICEBP) teóricas, basadas en la ecuación ( $8^{\prime}$ ) para México y Argentina, son 2.1 y 1.7 respectivamente. La interpretación de estas elasticidades es simple; por ejemplo, México tiene una EIICEBP igual a 2.1 resultado de la relación entre la tasa de crecimiento de las exportaciones y la del ingreso, por tanto si la elasticidad verdadera fuera mayor a 2.1 cuando la economía comienza a crecer las importaciones tenderían a crecer más rápidamente que las exportaciones y por tanto se generaría un desequilibrio de la BP que generaría una necesidad de financiamiento externo, con una pérdida de reservas internacionales si hay un tipo de cambio nominal fijo o con una depreciación real del tipo de cambio si hay un tipo de cambio nominal flexible. Ninguna de las tres soluciones podría mantenerse de forma permanente. De esta manera la economía no alcanza el pleno empleo y por tanto entra en un círculo vicioso donde las posibilidades de crecimiento se ven truncadas.

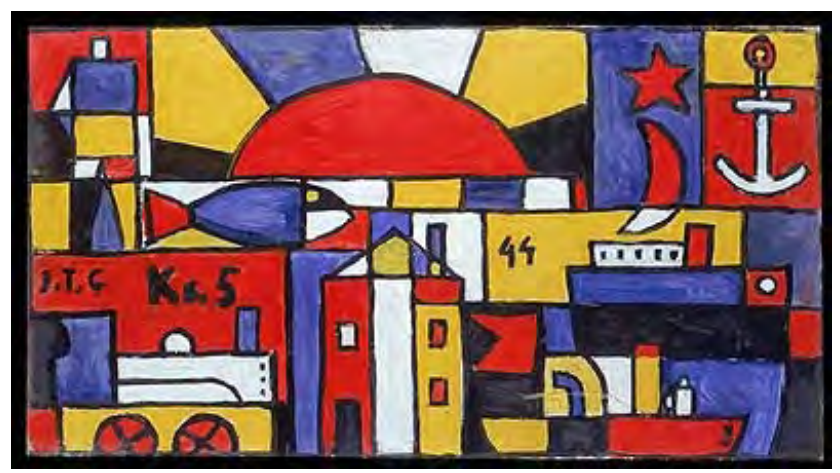


Tabla 1. Resultados de las estimaciones de las elasticidades ingreso de las importaciones.

\begin{tabular}{|c|c|c|c|c|}
\hline & \multicolumn{2}{|c|}{ México } & \multicolumn{2}{|c|}{ Argentina } \\
\hline Tasas de crecimiento & $\dot{x}_{t}$ & $\dot{y}_{t}$ & $\dot{x}_{t}$ & $\dot{y}_{t}$ \\
\hline 1960-2014 & 8.2 & 3.9 & 4.6 & 2.7 \\
\hline $2003-2014$ & 4.4 & 2.3 & 2.0 & 4.8 \\
\hline \multicolumn{5}{|c|}{ Elasticidades } \\
\hline \multicolumn{5}{|l|}{ 1.- LT-original } \\
\hline$\hat{\varepsilon}_{1960-2014}$ & \multicolumn{2}{|c|}{2.1} & \multicolumn{2}{|c|}{1.7} \\
\hline$\hat{\varepsilon}_{2003-2014}$ & \multicolumn{2}{|c|}{1.9} & \multicolumn{2}{|c|}{0.4} \\
\hline \multicolumn{5}{|l|}{ 2.- LT-Flujos de Capital } \\
\hline$\hat{\varepsilon} F C_{1960-2014}$ & \multicolumn{2}{|c|}{2.0} & \multicolumn{2}{|c|}{1.6} \\
\hline \multicolumn{5}{|c|}{ 3.- Elasticidades Implícitas } \\
\hline Sin Constante $\Omega$ & \multicolumn{2}{|c|}{$1.0^{* * *}$} & \multicolumn{2}{|c|}{$1.1^{* * *}$} \\
\hline Constante $^{\Omega}$ & \multicolumn{2}{|c|}{3.4} & \multicolumn{2}{|c|}{$2.5^{* * *}$} \\
\hline \multicolumn{5}{|c|}{ 4.- Función de demanda por importaciones } \\
\hline $1960-2014^{\Omega}$ & \multicolumn{2}{|c|}{$2.1^{* * *}$} & \multicolumn{2}{|c|}{$2.4^{* * *}$} \\
\hline $2003-2014^{\Omega}$ & \multicolumn{2}{|c|}{$2.4^{* * *}$} & \multicolumn{2}{|c|}{$1.5^{* * *}$} \\
\hline $\begin{array}{l}\text { Prueba de Hipótesis } \\
\text { restringiendo el valor } \\
\text { de } \pi\end{array}$ & \multicolumn{2}{|c|}{$\pi=2.1$} & \multicolumn{2}{|c|}{$\pi=1.7$} \\
\hline Chi_Cuadrado & \multicolumn{2}{|c|}{0.0001} & \multicolumn{2}{|c|}{6.68} \\
\hline Valor de $p$ & \multicolumn{2}{|c|}{$0.99^{* * *}$} & \multicolumn{2}{|c|}{0.01} \\
\hline \multicolumn{5}{|c|}{ Tasas de crecimiento -Johansen (1995)- } \\
\hline 1960-2014 & \multicolumn{2}{|c|}{3.9} & \multicolumn{2}{|c|}{2.0} \\
\hline 2003-2014 & \multicolumn{2}{|c|}{1.8} & \multicolumn{2}{|c|}{1.3} \\
\hline \multicolumn{5}{|c|}{ Diferencia entre la tasa de crecimiento efectiva y (\%) } \\
\hline 1960-2014 & \multicolumn{2}{|c|}{$-0.1 \%$} & & \\
\hline 2013-2014 & & & & \\
\hline
\end{tabular}

Fuente: elaboración propia.

Referencias: $x_{t}$ tasa de crecimiento exponencial de las exportaciones reales en el periodo de referencia. $y_{\text {t }}$ tasa de crecimiento del PIB en el periodo de referencia. $y_{t}$ ver la ecuación (8'). ${ }^{* * t}$ Significativa al $1 \% .{ }^{\Omega}$ Los errores del modelo no presentan autocorrelación ni heterocedasticidad, las pruebas de diagnóstico están disponibles bajo pedido al autor.

La situación es diferente cuando la elasticidad efectiva es menor a la EIICEBP. En este caso si la economía crece la dinámica de la BP no la restringe, ya que las importaciones van a crecer a una tasa menor a la que podrían hacerlo dada la relación entre las elasticidades ingreso y la tasa de crecimiento mundial. Nótese que, en este modelo, en ninguno de los dos casos se explica cómo la economía alcanza el equilibrio una vez que se desvía de la tasa de crecimiento consistente con el equilibrio de la balanza de pagos. 
Siguiendo a McCombie (1997) se investiga si existe o no cointegración entre los volúmenes de producción y los de exportación, en caso positivo respaldaría los principios detrás de la ley de Thirlwall. Para ello, en cada una de las tres economías se calcula un modelo vectorial de corrección de errores (VEC) para el periodo 1960-2014, con datos del PIB y las exportaciones reales expresadas a precios constantes, con datos anuales del Banco Mundial. Para el cálculo de los modelos primero se estableció el orden de integración de las variables, el número óptimo de rezagos a tener en cuenta para el ajuste de corto plazo del modelo, luego se calcularon las pruebas correspondientes para establecer el número de vectores de cointegración (ver el Anexo donde se encuentran todas las pruebas relevantes que se presentan en esta sección).

El valor estimado de la elasticidad se presenta en la tabla 1. Las pruebas de la traza y el máximo valor propio indican que existe un vector de cointegración cuando se supone que el mismo tiene una constante, como cuando se supone lo contrario. Por tanto, el primer resultado importante es que el PIB y las exportaciones tienen la misma tendencia estocástica. El resultado más robusto econométricamente es el modelo sin constante, normalizado para el PIB. En el modelo se usaron variables en logaritmos naturales; por tanto, el coeficiente normalizado de las exportaciones indica que en el largo plazo la elasticidad es unitaria. El movimiento conjunto de estas dos variables nos indica el cumplimiento de una de las implicaciones más importantes de la ley de Thirlwall: en el largo plazo la balanza de pagos debe mantener su equilibrio y ello en términos de tasa de crecimiento implica que las exportaciones y las importaciones deben crecer a la misma velocidad. Por tanto, partiendo de la ecuación ( $\left.8^{\prime}\right)$, es decir $y_{t}^{B O P^{\prime}}=\frac{1}{\pi} x_{t}$, y teniendo en cuenta que $\pi=\frac{m}{y}$, lo que dice la Ley de Thirlwall es que en el largo plazo $x=m$. Los datos de la tabla 1 respaldan la intuición detrás de la LT. Cuando el modelo se calculó con una constante en el vector de cointegración, se encontraron resultados relevantes solo para Argentina.

A continuación, se presentan los resultados del modelo de cointegración de la función de demanda de importaciones de la ecuación (2). En el Anexo se muestran las pruebas pertinentes del modelo. Es importante mencionar que tanto las importaciones como el PBI resultaron variables con un grado de integración unitario de acuerdo con las pruebas tradicionales (Dickey y Fuller DGLS y Phillip Perron) para los dos países; en cambio, los tipos de cambio real de México y Argentina resultaron ser variables estacionarias -I(0)-. Debido a que las pruebas de raíz unitaria tienden a identificar como variables I(1) a variables estacionarias que presentan algún tipo de inestabilidad estructural, se procedió a estimar pruebas de raíz unitaria que tuvieran en cuenta este aspecto. Los tipos de cambios reales en 
Argentina y México pueden considerarse tanto estacionarios o que tienen una raíz unitaria según el tipo y la cantidad de cambios estructurales que se consideren'; debido a ello se procedió de la siguiente manera: primero se aplicó la metodología de Johansen (1995) y se consideró que todas las variables presentaban una raíz unitaria en cada una de las economías consideradas. Luego se procedió a calcular vectores de cointegración a través de modelos ARDL, los cuales incorporan la posibilidad de que una o más de las variables en un modelo de cointegración sean estacionarias ${ }^{3}$.

En la tabla 1 se muestran las elasticidades ingreso de la demanda de importaciones, calculadas a través de la metodología de Johansen (1995). Para México se confirman los resultados obtenidos previamente, ya que muestra que en el largo plazo una variación del 1 \% en el PIB genera una variación de las importaciones de $2.1 \%$. Nótese que las altas elasticidades solo son posibles en economías donde se produce un proceso de apertura considerable, como es el caso de las dos economías analizadas. El índice de apertura comercial (exportaciones más importaciones sobre PIB) de las economías que estamos estudiando presenta un crecimiento notable en el periodo de estudio, que se muestra en la gráfica 1.

Gráfico 1. Apertura comercial medida en términos reales, 1960-2014.

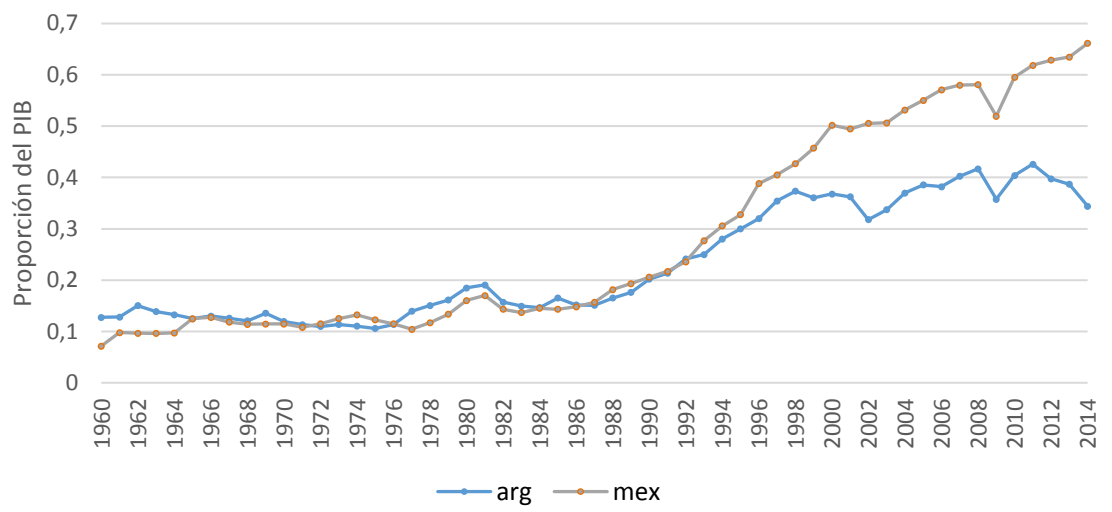

Fuente: Indicadores del desarrollo del Banco Mundial. Referencias: arg: Argentina y mex: México.

El indicador se calcula como la suma de las exportaciones y las importaciones dividida por el producto bruto interno. 
El caso de México es llamativo, ya que partiendo de un nivel de apertura similar al de Argentina en los años 1960 y 1970 en la actualidad el comercio exterior representa $66 \%$ del PIB medido en valores reales. En la gráfica 1 la trayectoria de las importaciones llama la atención; en tanto en 1987 habían alcanzado un mínimo histórico de $6 \%$ del PIB y a partir del siguiente año y a consecuencia de la aceleración del proceso de apertura comercial presentan un crecimiento continuo y en los años 2010 superan el $32 \%$ del PIB. Debido a este cambio notorio se incorporó una variable exógena dicotómica en el modelo de corrección de errores que toma el valor 1 desde 1987 hasta 2014 y 0 entre 1960 y 1986. La misma resultó significativa. Se trató de estudiar si hubo cambio estructural, pero no se pudo confirmar la hipótesis. Este resultado puede responder a que no se cuenta con la cantidad de datos suficientes y ello dificulta el estudio de cambio estructural. Debido a lo anterior se procedió a estimar modelos con datos trimestrales con la desventaja que abarcan un número menor de años. Los resultados se presentan más adelante.

Siguiendo a McCombie (1997:355) y Moreno (2003:349) se estimaron modelos de cointegración, utilizando los valores de las elasticidades ingreso de las importaciones calculadas aritméticamente como restricción para los valores de los coeficientes. De esta forma se estimaron pruebas de hipótesis, con el objetivo de observar si las mismas son una buena aproximación de las elasticidades estimadas con los modelos de la demanda de importaciones. Para México se aceptó la hipótesis que las elasticidades no son diferentes a las estimadas a través de la LT original al $1 \%$ de significancia (ver tabla 1). Con este resultado se puede afirmar que en estos casos pareciera que la TCCEBP que surge del modelo original de Thirlwall (1979) es una buena aproximación de la tasa de crecimiento efectiva. Es dable mencionar que en el caso de México las tasas de crecimiento calculadas a través de la ecuación ( $8^{\prime}$ ) y (12) son iguales, indicando que en el periodo de estudio el pago de intereses de la deuda externa no tuvo un impacto negativo sobre el desenvolvimiento de las economías analizadas. Es decir, si bien el pago de los intereses de la deuda externa tuvo un impacto negativo importante en los años 1980 y 1990, en términos del largo plazo parecieran no ser tan relevantes. Lo que tendría que observarse es una caída mayor en la elasticidad ingreso de la demanda de importaciones. Sin embargo, en el caso de México solo disminuye $5 \%$.

La tabla 1 muestra que el caso de Argentina es distinto al de México. La elasticidad ingreso de la demanda por importaciones es igual a 2.4; por tanto, un incremento del PBI en $1 \%$ genera un incremento de las cantidades importadas de $2.4 \%$ en el largo plazo. Mientras que la elasticidad teórica calculada a través de la LT original es igual a 1.7; lo que implica que ante un incremento del producto del $1 \%$ las importaciones 
pueden crecer $1.7 \%$ para mantener la BP en equilibrio. La diferencia entre las elasticidades en Argentina indica que los problemas de balanza de pago son más graves que en México. Para confirmar la diferencia entre las elasticidades se realizó la prueba de cointegración, suponiendo que la elasticidad de la demanda por importaciones era 1.7 no se aceptó a los niveles de significancia regulares. Un reflejo de esta diferencia puede observarse en la gráfica 2, la cual muestra la evolución de la deuda externa en las economías analizadas. Argentina muestra la mayor volatilidad, lo que indica la gran dificultad que ha tenido esta economía para financiar los desequilibrios de la cuenta corriente.

Gráfico 2. Evolución de la deuda externa con relación al PIB (\%), 1970-2014.

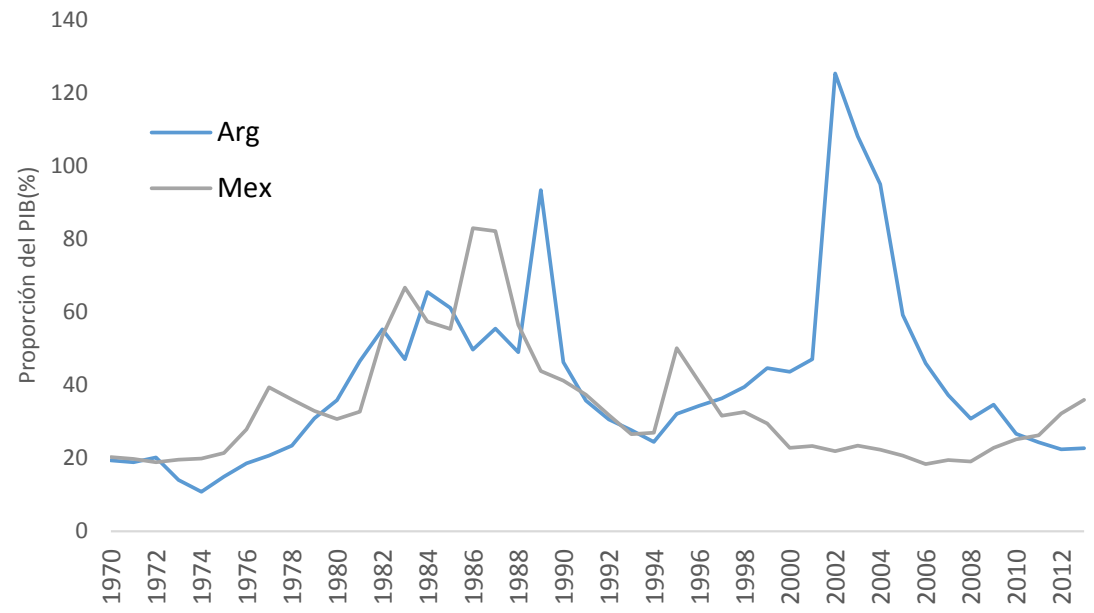

Fuente: Indicadores del desarrollo del Banco Mundial. Referencias: arg: Argentina y mex: México.

La gráfica 3 muestra la ratio entre la tasa de crecimiento efectiva y la tasa de crecimiento estimada a través de la ecuación (8') con las elasticidades ingreso de las demandas por importaciones calculadas en los modelos de cointegración. Para los años 1980 y principios de los 1990, la LT original no es una buena aproximación de la tasa de crecimiento efectiva. Específicamente, las estimaciones subestiman la tasa crecimiento de las economías. El modelo sugiere que este resultado se puede obtener si las elasticidades ingreso de la demanda de importación eran más bajas en esos años, el tipo de cambio real permitió un crecimiento mayor o se recibieron capitales del exterior para financiar el consumo interno o una desaceleración de las exportaciones. Desde el punto de vista de la LT, lo más probable es que el efecto se origine por un cambio estructural de las elasticidades. 
Gráfico 3. Ratio de la tasa de crecimiento consistente con el equilibrio de la BP y la tasa de crecimiento efectivo.

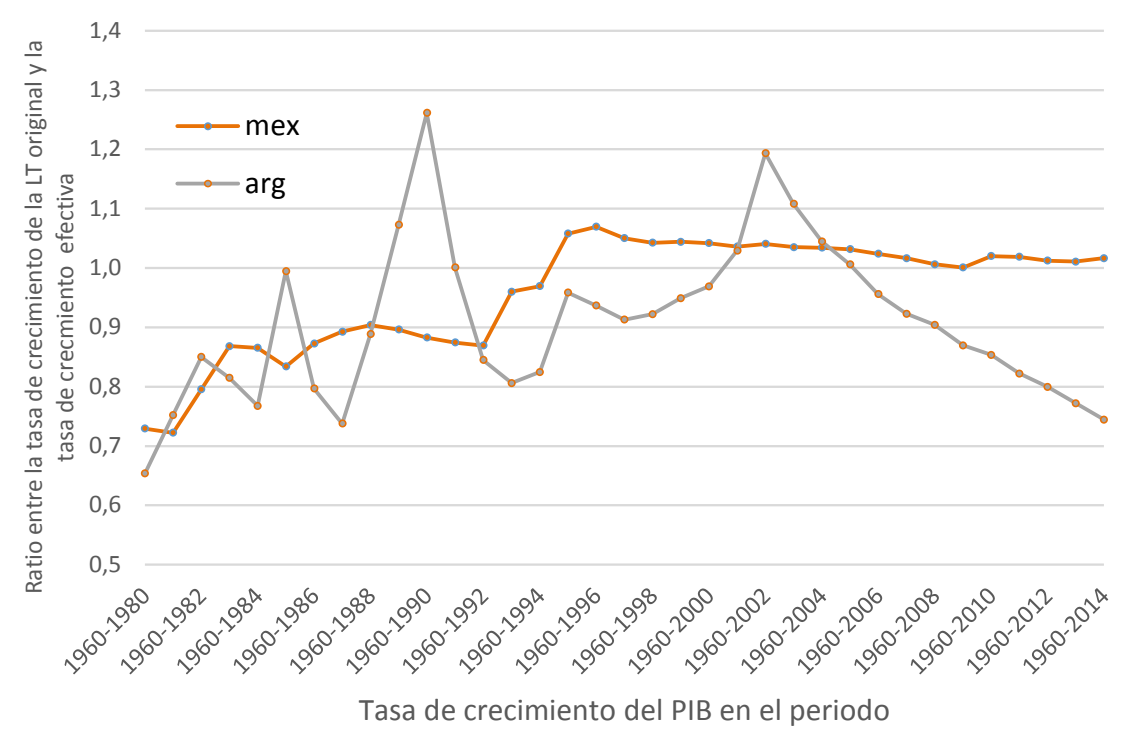

Fuente: elaboración propia. Referencias: arg: Argentina y mex: México.

En el caso de México, a partir de fines de los años 1990 las dos tasas de crecimiento convergen. Por tanto, se puede inferir que en el largo plazo la LT es una buena aproximación de la tasa de crecimiento efectiva. El caso de Argentina es diferente. Como se observa en la gráfica 3, mantiene una evolución similar a la de México hasta 2002. A partir de ese año la tasa de crecimiento estimada a través de la LT original y la efectiva empiezan a divergir, tendencia que se mantiene hasta el fin del periodo de estudio. Es interesante considerar dos explicaciones para esta performance; primero, una baja tasa de crecimiento de las exportaciones; y segundo, una aceleración de la tasa de crecimiento efectiva. De acuerdo a Thirlwall (1979) es improbable que los países financian continuamente un proceso de crecimiento por encima de la tasa de crecimiento consistente con la balanza de pagos. En el caso de Argentina, durante 14 años creció por encima de la tasa prescripta por la LT gracias a una mejora notable de los términos del intercambio (Rodrik, 2008; Ros, 2013); asimismo, en el periodo 2009-2014 se financió la divergencia con pérdidas de reservas internacionales.

En términos de las diferencias entre las economías se observa que México presenta tasas de crecimiento que coinciden con la tasa de crecimiento consistente con la LT. México al tener una elasticidad ingreso de las importaciones alta debe realizar un esfuerzo exportador mayor, creciendo sus envíos al exterior al $8.2 \%$ para alcanzar una tasa de crecimiento de 
$3.9 \%$. Argentina presenta una tasa de crecimiento menor ya que se encuentra en el peor de los escenarios, en tanto logró en el periodo de estudio una baja tasa de crecimiento de sus exportaciones y a la vez cuenta con una elevada elasticidad ingreso de las importaciones (2.4). Por tanto, los fundamentos de la LT ofrecen elementos para comprender las diferencias en las tasas de crecimiento entre estas dos economías analizadas en el periodo 1960-2014.

Sin embargo, en el corto plazo la LT pareciera no ser una buena aproximación de la tasa de crecimiento efectiva. La tabla 1 muestra la tasa de crecimiento que surge de la LT original en el periodo 20032014, calculada con las elasticidades estimadas en los modelos de cointegración del periodo 1960-2014. El caso de Argentina es el más obvio y la divergencia entre las tasas de crecimiento claramente se explica por las causas mencionadas más arriba. Sin embargo, en el caso de México las diferencias entre la tasa de crecimiento pronosticada a través de la LT y la efectiva también son significativas. Señalando que la prognosis es que en el largo plazo esta economía verá disminuir su tasa de crecimiento, convergiendo a la tasa de crecimiento consistente con la LT. Es decir, manteniendo las elasticidades ingreso estimadas, las tasas de crecimiento de las exportaciones en el periodo 2003-2014 implican una tasa de crecimiento menor a la del periodo 1960-2014.

Se estimaron modelos con datos trimestrales para el periodo 2003-2014 para estudiar el valor de la elasticidad ingreso de la demanda en ese periodo y analizar las diferencias con los resultados del modelo para el periodo de estudio completo. Los resultados se muestran en la tabla 1. En el caso de México se incrementó la elasticidad ingreso de las importaciones de 2.1 a 2.4. Cuando se compara las elasticidades estimadas con las calculadas a través de la LT original, obtenemos que en el caso de México la diferencia es de 1.3 veces. Mientras que para todo el periodo eran iguales. Es decir, según estos resultados, los problemas de crecimiento de esta economía se han agudizado en los últimos años, ya que, para una misma tasa de crecimiento de las exportaciones, la tasa de crecimiento consistente con el equilibrio de la balanza de pagos es menor.

El caso de Argentina es diferente porque la elasticidad ingreso de la demanda por importaciones disminuyó en el periodo 2003-2014 respecto al periodo ampliado, pasó de 2.4 a 1.5. La disminución puede reflejar un cambio positivo en la estructura productiva que posibilite el crecimiento de largo plazo. En términos estrictamente de la LT el cambio no pareciera ser significativo para resolver el problema de la restricción externa. En tanto, para el periodo 1960-2014 la diferencia entre la elasticidad efectiva y la teórica era 1.4 veces, mientras que en el periodo 
2003-2014 ese indicador se incrementó a 3.6, a pesar de la caída en la elasticidad ingreso de la demanda por importaciones los problemas de restricción externa parecen haberse profundizado en Argentina. Lo que implica que en el periodo 2003-2014 la tasa de crecimiento consistente con el equilibrio de la BP disminuyó a $1.3 \%$. A pesar de esta disminución, la economía argentina en este periodo presentó una aceleración en su tasa de crecimiento de $2.7 \%$ en el periodo completo a $4.8 \%$.

En el caso de Argentina, la diferencia entre la TCCEBP y la tasa de crecimiento efectiva en el periodo 2003-2014 es notable. La economía argentina creció por encima de la TCCEBP, debido al marcado incremento de los términos del intercambio en ese periodo, lo que permitió mantener el nivel de las exportaciones por encima de las importaciones medidas en dólares. Sin embargo, el proceso de crecimiento no modificó las elasticidades ingreso de las importaciones y las exportaciones ${ }^{2}$, por tanto $-\mathrm{y}$ siguiendo la lógica del modelo de Thirlwall- la ventaja inicial de la mejora en los términos del intercambio y el tipo de cambio real se fue diluyendo y en 2010 el otrora superávit de la cuenta corriente se convirtió en un déficit comercial. Hacia el final del periodo el proceso de deterioro de la BP se agudizó por la apreciación real del peso. En este contexto el Gobierno optó por implementar controles de capital que, en cierta medida, afectaron el crecimiento económico (Manzanelli et al., 2015:57). Por tanto, los precios relativos juegan un papel relevante en el corto plazo para evitar o agudizar los problemas que plantea la LT. Sin embargo, en el largo plazo la única forma que tiene Argentina para incrementar la TCCEBP y evitar los problemas de balanza de pagos es modificar la estructura productiva de la economía para procurar incrementar la elasticidad ingreso de las exportaciones y disminuir la elasticidad ingreso de las importaciones.

\section{Conclusiones}

Se puede afirmar que después de más de treinta años de haber sido propuesta la ley de Thirlwall sigue siendo un instrumento útil para analizar el desenvolvimiento de una economía en el tiempo o para explicar las diferencias de crecimiento entre economías. En los casos analizados en el presente trabajo queda establecido su poder explicativo en el largo plazo. En particular, su relevancia emerge cuando las economías aceleran su crecimiento como ha sido el caso de Argentina en el periodo 2003-2014, no así para México que presentó un crecimiento exiguo; en este caso el trabajo señala cuáles serían los obstáculos que debería sortear si la tasa de crecimiento de ese país se acelerara en el futuro. Es decir, para la economía 
mexicana la ley de Thirlwall se presenta como condición a superar y no como un modelo que explique las causas últimas de su lento crecimiento.

Los ejercicios teóricos y econométricos realizados en este artículo no deben eclipsar su contenido social y político. El mensaje que nos deja el modelo y los resultados estadísticos alcanzados es que para que las economías de América Latina puedan mantener una senda de crecimiento consistente con el pleno empleo y con el equilibrio de la balanza de pagos, las mismas deben incrementar la elasticidad ingreso de la demanda por importaciones y disminuir la elasticidad ingreso de la demanda por importaciones. La forma de alcanzar esos objetivos es complejizando las canastas de bienes exportados y disminuyendo la dependencia tecnológica con el exterior. Estos cambios en la estructura económica implican grandes acuerdos entre el Estado, los capitalistas y los trabajadores organizados. Los procesos de modernización que se deben llevar adelante suelen afectar a los trabajadores negativamente; por eso el Estado tiene que tener un rol clave que implique comandar estos procesos, procurando que los acuerdos alcanzados beneficien a todos los ciudadanos de la economía especialmente a los trabajadores.

\section{Referencias Bibliográficas}

- ABELES, Martín, LAVARELLO, Pablo y MONTAGU, Haroldo. (2013). Heterogeneidad estructural y restricción externa en la economía argentina. En: INFANTE, Ricardo y GERSTENFELD, Pascual, eds. Hacia un desarrollo inclusivo: el caso de la Argentina. Santiago de Chile-CEPAL, OIT.

- $\quad$ ACEMOGLU, Daron \& ROBINSON, James. (2012). Why Nations Fail? The origins of power, prosperity, and poverty. Crown Business, Nueva York-Crown Business

- $\quad$ AMICO, Fabián, FIORITO, Alejandro y HANG, Guillermo. (2011). Producto potencial y demanda en el largo plazo: hechos estilizados y reflexiones sobre el caso argentino reciente. CEFID-AR. Documento de trabajo N`35, enero, 2011. Buenos Aires-CEFID-AR

- CALDENTEY, Esteban. (2015, abril-junio). Una lectura crítica de 'la lectura crítica' de la Ley de Thirlwall. En: Investigación Económica, LXXIV(292), 47-65. Ciudad de México-UNAM.

- CALDENTEY, Esteban. (2016). A time to reflect on opportunities for debate and dialogue between (neo)structuralism and heterodox schools of thought. En: BÁRCENA, Alicia y PRADO, Antonio, eds. Neostructuralism and heterodox thinking in Latin America and the Caribbean in the early twenty-first century. Santiago de Chile-CEPAL.

- CLAVIJO, Pedro y ROS, Jaime (2015, abril-junio). La Ley de Thirlwall: una lectura crítica. En: Investigación Económica, LXXIV(292), 11-40. Ciudad de México-UNAM

- FURTADO, Celso. (1956). Manual of Programming and Economic Development Promotion. Nueva York- Naciones Unidas.

- IBARRA, Carlos. (2015, abril-junio). Comentario a "La Ley de Thirlwall: una lectura crítica" de Pedro Clavijo y Jaime Ros. En: Investigación Económica, LXXIV (292), 4145. Ciudad de México-UNAM. 
- JOHANEN, Søren. (1995). Likelihood-Based Inference in Cointegrated Vector Autoregressive Models. Oxford University Press. Oxford.

- KEMP, Murray. (2008). Marshall-Lerner condition. En:The New Palgrave Dictionary of Economics. Segunda edición. Eds.: S. N. Durlauf y L. E. Blume. Nueva YorkPalgrave Macmillan

- MANZANELLI, Pablo, BARRERA, Mariano, WAINER, Andrés y BONA, Leandro (2015, abril). Deuda externa, fuga de capitales y restricción externa. Desde la última dictadura militar hasta la actualidad. CEFID-AR. Documento de Trabajo № 68. Buenos Aires-CEFID-AR.

- McCOMBIE, John. (1997). On the empirics of balance-of-payments-constrained growth. En: Journal of Post Keynesian Economics, 19, 345-75. Nueva York-LondresTaylor y Francis.

- MORENO, Juan y ROS, Jaime. (2010). Desarrollo y crecimiento en la economía mexicana. Una perspectiva histórica. Ciudad de México-Fondo de Cultura Económica.

- MORENO, Juan. (2003). Capital Flows, Interest Payments and The BalanceofPayments Constrained Growth Model: A theoretical and Empirical Analysis. En: Metroeconomica, 54(2 \& 3), 346-365. Roma-John Wiley e Hijos Ltd.

- $\quad$ PERROTINI, Ignacio (2002, jul-dic). La ley de Thirlwall y el crecimiento en la economía global: análisis crítico del debate. En: Revista Venezolana de Análisis de Coyuntura, VIII(2), 117-141. Caracas-Universidad Central de Venezuela.

- PREBISCH, Raúl (1949). El desarrollo económico de la América Latina y algunos de sus principales problemas. En: Desarrollo Económico, 26(103). (Oct - Dec., 1986), 479-502. Buenos Aires-Universidad De Buenos Aires.

- RODRIK, Andy. (2008). The real exchange rate and economic growth. Brookings Papers on Economic Activity, 2, 365-412. Nueva York-BP.

- ROS, Jaime, (2013). Latin America's Trade and Growth Patterns, the China Factor, and Prebisch's Nightmare. En: Journal of Globalization and Development. Nueva York-Banco Mundial.

- THIRLWALL, Anthony (2003). La naturaleza del crecimiento económico. Un marco alternativo para comprender el desempeño de las naciones. Fondo de Cultura Económica, Ciudad de México, 2003.

- THIRLWALL, Anthony y HUSSAIN, Nureldin (1982). The Balance of Payments Constraint, Capital Flows and Growth Rate Differences between Developing Countries, Oxford Economic Papers, 34(3), 498-510. Londres-Oxford University Press.

- THIRLWALL, Anthony. (1979). The balance of payments constraint as an explanation of the international growth rate differences, En: Banca Nazionale del Lavoro Quarterly Review, 128, 45-53. Roma-Banca Nazionale del Lavoro.

- VERNENGO, Matías. (2015). Una lectura crítica de la crítica al modelo de Thirlwall. En: Investigación Económica, LXXIV (292), 67-80. Ciudad de México-Universidad Nacional Autónoma de México.

Para citar este artículo:
Capraro, S.G.M. (2017). La Ley de Thirwall: teoría y evidencia empírica. Los casos de México y Argentina en el período 19602014. Teuken Bidikay Vol. 8 N¹1. Pp. 99-118 


\section{Anexo:}

Tabla A.1. Pruebas de raíces unitarias: Dickey-Fuller-GLS y Phillip-Perron.

\begin{tabular}{|c|c|c|c|c|c|}
\hline Var & $\begin{array}{c}\text { Criterio } \\
\text { de Información }\end{array}$ & $\begin{array}{l}\text { Rezagos } \\
\text { óptimos }\end{array}$ & $\begin{array}{c}\text { Estadístico } \\
\text { DF-GLS }\end{array}$ & $\begin{array}{c}\text { Valor Crítico } \\
\text { al } 5 \%\end{array}$ & OI \\
\hline \multicolumn{6}{|c|}{ México 1962-2014 } \\
\hline X_mx & SC/MAIC & 1 & -2.687 & -3.202 & 1 \\
\hline Y_mx & SC/MAIC & 1 & -0.621 & -3.202 & 1 \\
\hline \multirow[t]{2}{*}{ M_mx } & SC & 1 & -2.779 & -3.202 & 1 \\
\hline & MAIC & 3 & -1.904 & -3.108 & 1 \\
\hline \multirow[t]{2}{*}{ Q_mx } & SC & 1 & -3.432 & -3.231 & 0 \\
\hline & MAIC & 2 & -2.268 & -3.182 & 1 \\
\hline \multicolumn{6}{|c|}{ Argentina 1962-2014 } \\
\hline \multirow[t]{2}{*}{ X_ar } & SC & 1 & -2.687 & -3.202 & 1 \\
\hline & MAIC & 2 & -1.996 & -3.159 & 1 \\
\hline Y_ar & SC/MAIC & 1 & -2.192 & -3.202 & 1 \\
\hline M_ar & SC/MAIC & 1 & -2.670 & -3.202 & 1 \\
\hline Q_ar & SC/MAIC & 1 & -2.630 & -3.209 & 1 \\
\hline
\end{tabular}

Fuente: Elaboración propia

Referencias: X: exportaciones, M: importaciones, Y: Producto Bruto Interno, Q: tipo de cambio real, Br: Brasil, mx: México, ar: Argentina. Ol: orden de integración según la prueba, SC: Criterio de información de Schwarz; MAIC: Criterio de información modificado de Akaike.

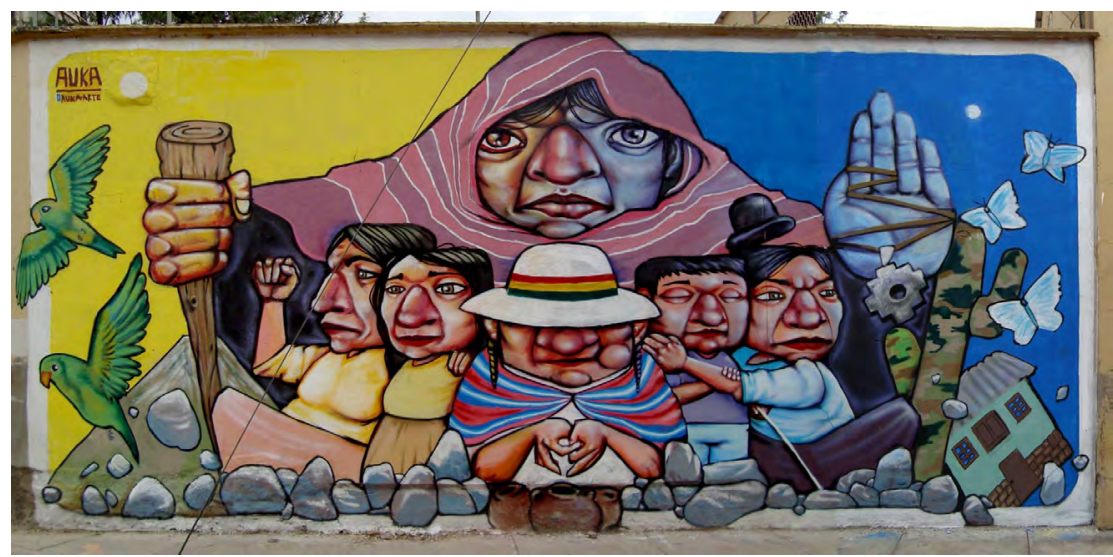


Tabla A.2. Pruebas de Cointegración

\begin{tabular}{|c|c|c|c|c|c|}
\hline $\begin{array}{l}\text { Número de vectores } \\
\text { de Cointegración }\end{array}$ & Valor propio & \begin{tabular}{|c|} 
Prueba \\
de la traza
\end{tabular} & $\begin{array}{c}\text { Valor Crítico } \\
\text { al } 5 \%\end{array}$ & \begin{tabular}{|l|} 
Valor \\
de P
\end{tabular} & NR \\
\hline \multicolumn{6}{|c|}{ México 1962-2014: X_mx y Y_mx + } \\
\hline \multicolumn{6}{|c|}{ Sin constante en el vector de cointegración } \\
\hline $0^{*}$ & 0.33 & 21.07 & 12.33 & 0.01 & 1 \\
\hline 1 & 0.02 & 0.6 & 4.13 & 0.51 & 1 \\
\hline \multicolumn{6}{|c|}{ Con constante en el vector de cointegración + } \\
\hline $0^{*}$ & 0.33 & 28.49 & 20.27 & 0.01 & 1 \\
\hline 1 & 0.14 & 7.53 & 9.17 & 0.11 & 1 \\
\hline \multicolumn{6}{|c|}{ Argentina 1962-2014: X_aryY_ar + } \\
\hline \multicolumn{6}{|c|}{ Sin constante en el vector de cointegración } \\
\hline $0^{*}$ & 0.24 & 16.5 & 12.33 & 0.01 & 1 \\
\hline 1 & 0.05 & 2.42 & 4.13 & 0.15 & 1 \\
\hline \multicolumn{6}{|c|}{ Con constante en el vector de cointegración } \\
\hline $0^{*}$ & 0.3 & 23.58 & 20.27 & 0.02 & 1 \\
\hline 1 & 0.1 & 5.29 & 9.17 & 0.26 & 1 \\
\hline \multicolumn{6}{|c|}{ México 1962-2014: M_mx, Y_mx, Q_mx + } \\
\hline $0^{*}$ & 32.61 & 24.28 & 0.01 & 0.36 & 1 \\
\hline 1 & 11.23 & 12.33 & 0.08 & 0.15 & 1 \\
\hline \multicolumn{6}{|c|}{ Argentina 1962-2014: M_ar, Y_ar, Q_ar ++ } \\
\hline $0^{*}$ & 0.35 & 30.07 & 29.8 & 0.05 & 1 \\
\hline 1 & 0.09 & 8.3 & 15.5 & 0.44 & 1 \\
\hline \multicolumn{6}{|c|}{ México 2003_q1-2014_q4: $M \_m x, Y \_m x, Q \_m x++$} \\
\hline $0^{*}$ & 0.41 & 36.4 & 29.8 & 0.01 & 9 \\
\hline 1 & 0.21 & 11.14 & 15.5 & 0.21 & 9 \\
\hline \multicolumn{6}{|c|}{ Argentina 2003_q1-2014_q4: M_ar, Y_ar, Q_r ++ } \\
\hline $0^{*}$ & 0.51 & 52.7 & 35.2 & 0.01 & 3 \\
\hline 1 & 0.28 & 18.64 & 20.27 & 0.09 & 3 \\
\hline
\end{tabular}

Fuente: Elaboración propia

Referencias: Para el nombre de las variables ver la tabla A.1. Nl: número de rezagos. + el modelo no considera una constante en el vector de cointegración, ++ el modelo considera una constante en el vector de cointegración. * Existe un vector de cointegración al 0.05 de significancia. 